\title{
Intravesical Therapy for Superficial Bladder Carcinoma
}

The adequate treatment for superficial bladder carcinoma with infiltration only in the mucosa (Ta) or submucosa (T1) is complete surgical removal by a single or repeated transurethral resection. It is well documented in many studies that $40-80 \%$ of such patients will have recurrent tumors. A minority of such recurrent tumors will show progression in grade and stage. Two reasons for recurrent tumor are discussed: multifocality due to field cancerization and implantation of single cells during transurethral resection.

Patients with a history of transitional cell carcinoma of the bladder can be easily segregated based on well-established risk factors. For example, patients with low-grade Ta transitional cell carcinoma are at little risk for progression and are primarily monitored for the development of a subsequent tumor. In addition to grade and stage, other well-established risk factors for recurrence and progression are multifocality, morphology of the tumor (papillary or solid), disease-free interval, and especially the result of the endoscopic examination 3 months after transurethral resection. The risk of developing progressive diseases is almost zero in TaGI tumors and can be as high as $48 \%$ in T1GIII tumors, especially when it is associated with carcinoma in situ.

Instillation of alkalizing chemotherapeutic agents have been shown to cure up to $50 \%$ of superficial bladder carcinoma, but also to reduce recurrence rate when instillations of the bladder are used for prophylaxis. There is a strong debate about the possibility to influence progression rate and survival by means of prophylactic chemotherapeutic instillations into the bladder. Immunotherapeutics, especially BCG, but also interferon, likewise are potent prophylactic and therapeutic agents when used for bladder instillations. The main question in the 1980s and 1990s was how long such a prophylactic schedule should be, and to define the exact indication. There has been a wide range of recommendations, e.g. life-long prophylaxis every 2 weeks versus one single shot in the first hours after transurethral resection. It seems now that there is some consensus that pTa tumors with low malignant potential, e.g. GI, have such a low progression rate that chemoprophylaxis or immunoprophylaxis is not warranted, except in the rare cases where multifocality is seen in the 3-month cystoscopy after trans- urethral resection (TUR) or when the tumor is accompanied by carcinoma in situ. In pTaGII, GIII, and pT1GI and GII tumors, especially when they are multifocal, chemoprophylaxis or immunoprophylaxis is recommended. In general, 6 weekly instillations of BCG, or one postoperative instillation of an alkalizing chemotherapeutic agent in the first hours after TUR, or a long-term prophylaxis for e.g. 6 months or longer with instillations every other week are given. My personal experience is that long-term prophylaxis using chemotherapeutic agents like doxorubicine or mitomycin $\mathrm{C}$ has some impact on progression rate. Recently, maintenance therapy is also recommended in those who received 6 weekly BCG instillations. However, a recent meta-analysis [1] revealed a 20-percentdecrease in the risk of tumor recurrence at 8 years of adjuvant instillation chemotherapy and no effect on the risk of progression. In a 15-year follow-up the group of the Memorial Sloan Kettering Cancer Center [2] found no significant difference in disease-specific survival in patients who received BCG. Many studies showed no difference in progression or survival comparing mitomycin $\mathrm{C}$ and BCG. This is an unsolved problem, especially because a high proportion of bladder cancer deaths among patients with progression is well documented. There is some evidence that biological markers that indicate the progression potential of a tumor like p53 protein accumulation might help us in the future [3].

\section{References}

1 Pawinski A, Sylvester R, Kurth KH, Bouffioux C, van der Meijden A, Parmar MK, Bijnens LA: Combined analysis of European Organization for Research and Treatment of Cancer, and Medical Research Council randomized clinical trials for the prophylactic treatment of stage TaT1 bladder cancer. European Organization for Research and Treatment of Cancer Genitourinary Tract Cancer Cooperative Group and the Medical Research Council Working Party on Superficial Bladder Cancer. J Urol 1996;156:934.

2 Cookson MS, Herr HW, Zhang ZF, Soloway S, Sogani PC, Fair WR: The treated natural history of high risk superficial bladder cancer: 15-Year outcome. J Urol 1997;158:62.

3 Fair W, Herr H, Reuter V: Nuclear overexpression of p53 protein in transitional cell bladder cancer: a marker for disease progression. J Natl Cancer Inst 1993;85:53.

H. Huland, Hamburg

\begin{tabular}{ll}
\hline KARGER & $\oplus$ 1999 S. Karger GmbH, Freiburg \\
Fax +49 761 4520714 & Accessible online at: \\
$\begin{array}{l}\text { E-mail kargergmbh@aol.com } \\
\text { www.karger.com }\end{array}$ & http://BioMedNet.com/karger
\end{tabular}

